\title{
Facile synthesis of benzothiadiazine 1,1-dioxides, a precursor of RSV inhibitors, by tandem amidation/ intramolecular aza-Wittig reaction
}

\author{
Krishna C. Majumdar ${ }^{\star} \S$ and Sintu Ganai
}

Open Access

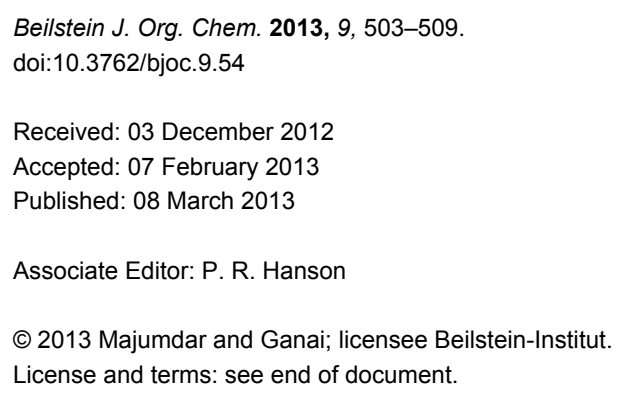

\begin{abstract}
Reaction of $o$-azidobenzenesulfonamides with ethyl carbonochloridate afforded the corresponding amide derivatives, which gave 3-ethoxy-1,2,4-benzothiadiazine 1,1-dioxides through an intramolecular aza-Wittig reaction. The reaction was found to be general through the synthesis of a number of benzothiadiazine 1,1-dioxides. Acid-catalyzed hydrolysis of 3-ethoxy-1,2,4-benzothiadiazine 1,1-dioxides furnished the 2-substituted benzothiadiazine-3-one 1,1-dioxides in good yields and high purity, which is the core moiety of RSV inhibitors.
\end{abstract}

\section{Introduction}

Sultams have gained popularity in the scientific community especially among synthetic and medicinal chemists, because this basic moiety is present in many natural products and biologically active substances [1-8]. Especially, benzothiadiazine3-one 1,1-dioxide and its derivatives possess potential activity, including hypoglycemic [9], anticancer and anti-HIV activity [10-13], and also serve as selective antagonists of $\mathrm{CXR}_{2}$ [14]. 2-Substituted-2H-1,2,4-benzothiadiazine-3(4H) one 1,1-dioxides showed varying degrees of sedative and hypotensive activities [15]. A number of benzothiadiazine 1,1-dioxide derivatives have recently been reported that display potent activity [16-22], including hypoglycemic (1), anti-HIV (2), HIV-1 specific non- nucleoside reverse transcriptase inhibitor (3), sedative (4), and respiratory syncytial virus (RSV) inhibitory activity (5; Figure 1).

A literature search revealed that the 1,2,4-benzothiadiazine 1,1dioxides are generally synthesized either by condensation of $o$-aminobenzenesulfonamides with urea at elevated temperature [23] or by the reaction of $o$-aminobenzenesulfonamide with isocyanates in DMF under reflux [24]. Although various approaches to the preparation of 1,2,4-benzothiadiazine 1,1dioxide derivatives have been reported [25-32], the development of a simpler method for the synthesis of the 1,2,4-benzo- 
<smiles>O=C1Nc2ccc(Cl)cc2S(=O)(=O)N1</smiles>

1

hypoglycemic agent<smiles>CN1C(=O)N(CCc2nc3ccccc3n2CCC(F)(F)F)c2ccccc2S1(=O)=O</smiles>

5<smiles>O=C1Nc2ccccc2S(=O)(=O)N1</smiles>

2

anti-HIV activity<smiles>CN1C(=O)N(Cc2ccccc2)S(=O)(=O)c2cscc21</smiles>

3

HIV-1 specific nonnucleoside reverse transcriptase inhibitor<smiles>[R]N1C(=O)Nc2ccccc2S1(=O)=O</smiles>

4

sedative

RSV inhibitor

Figure 1: Biologically active 1,2,4-benzothiadiazine 1,1-dioxide derivatives.

thiadiazine 1,1-dioxide moiety is still desirable because of their biological significance.

The aza-Wittig reaction is employed for the construction of $\mathrm{C}=\mathrm{N}, \mathrm{N}=\mathrm{N}$ and $\mathrm{S}=\mathrm{N}$ double bonds in various heterocycles and heterocycle-containing natural products [33-43]. Recently, we have synthesized asymmetrically substituted piperazine-2,5dione derivatives using the intramolecular aza-Wittig reaction [44]. In continuation of our earlier work [45-51], we have undertaken a study to synthesize 1,2,4-benzothiadiazine 1,1dioxide derivatives using an intramolecular aza-Wittig reaction as the key step. Herein we report our results.

Retrosynthetic analysis of the RSV inhibitors 5 and $\mathbf{6}$ relied on benzothiadiazine-3-one 1,1-dioxide 7, which can easily be obtained by simple hydrolysis of the benzothiadiazine 1,1dioxide derivative $\mathbf{8}$. Construction of this six-membered sultam 8 was thought to be achieved by intramolecular aza-Wittig reaction of the $o$-azido derivative 9. The following retrosynthetic analysis led us to the starting material $o$-azidobenzenesulfonic acid (11) for the synthesis of the intermediate $\mathbf{1 0}$ necessary for the synthesis of RSV inhibitors (Scheme 1).

\section{Results and Discussion}

Sulfonic acid $\mathbf{1 1}$ bearing an $o$-azido group [30] was converted into the corresponding sulfonyl chloride by treatment with oxalyl chloride followed by the reaction with appropriate amines to give the requisite 2-azido- $N$-substituted benzenesulfonamides 10a-i. The sulfonamide $\mathbf{1 0 b}$ was reacted with ethyl carbonochloridate to afford the corresponding amide derivative

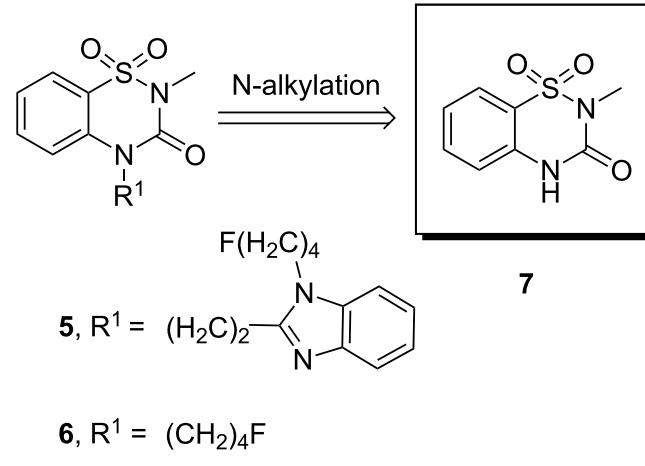

RSV inhibitors<smiles>[R]OC(=O)N(C)S(=O)(=O)c1ccccc1N=CC=CC=C</smiles><smiles>Nc1ccccc1S(=O)(=O)O</smiles>

11<smiles>[CH]</smiles><smiles>[R]C(=O)OC(=[X])C</smiles> 
9b required for our study. Initially, we turned our attention to the synthesis of a benzothiadiazine 1,1-dioxide derivative using substrate $9 \mathbf{b}$ by intramolecular aza-Wittig reaction. To test this premise, 9b was treated with triphenylphosphine in THF at room temperature, but no desired product was obtained, and only the intermediate iminophosphorane $\mathbf{1 2 b}$ was isolated, even under reflux (Scheme 2).

We next conducted a series of reactions with the replacement of the solvent THF by other solvents, such as toluene, $\mathrm{CH}_{2} \mathrm{Cl}_{2}$, and $\mathrm{CH}_{3} \mathrm{CN}$, but none of them afforded any cyclized product (Table 1, entries 2-4,). Then the reaction conditions were modified through the use of a higher-boiling-point solvent, i.e., $o$-dichlorobenzene (DCB). The reaction was successful at higher temperature, affording the desired cyclized product $\mathbf{1 3 b}$ (54\%) along with the by-product triphenylphosphine oxide (Table 1 , entry 5).

Subsequently, we turned our attention to develop a simpler onestep procedure by heating the sulfonamide $\mathbf{1 0 b}$ with ethyl carbonochloridate, $\mathrm{Et}_{3} \mathrm{~N}$ and $\mathrm{PPh}_{3}$ in $\mathrm{DCB}$ at $135^{\circ} \mathrm{C}$ for $6 \mathrm{~h}$, which gave the cyclized product $\mathbf{1 3 b}$ in $78 \%$ yield (Table 2, entry 1). The base $\mathrm{Et}_{3} \mathrm{~N}$ was then replaced by $\mathrm{Cs}_{2} \mathrm{CO}_{3}$ or $\mathrm{K}_{2} \mathrm{CO}_{3}$, but no better result was obtained (Table 2, entries 2 and 3). Only DIPEA gave $69 \%$ yield of the product (Table 2, entry 4). However, surprisingly the use of xylene as the solvent improved the yield of the cyclized product (Table 2, entry 5). The replacement of $\mathrm{NEt}_{3}$ by DIPEA as the base also gave a similar

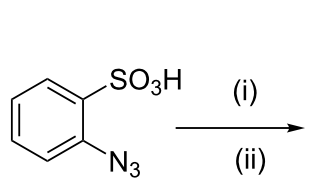

11 (ii)<smiles>Nc1ccccc1S(=O)(=O)O</smiles>

10 10a, $\mathrm{R}=\mathrm{C}_{6} \mathrm{H}_{5}$

10b, $\mathrm{R}=4-\mathrm{Cl}-\mathrm{C}_{6} \mathrm{H}_{4}$

10c, $\mathrm{R}=4-\mathrm{COCH}_{3}-\mathrm{C}_{6} \mathrm{H}_{4}$

10d, $\mathrm{R}=4-\mathrm{CO}_{2} \mathrm{CH}_{3}-\mathrm{C}_{6} \mathrm{H}_{4}$

10e, $\mathrm{R}=4-\mathrm{CH}_{3}-\mathrm{C}_{6} \mathrm{H}_{4}$

10f, $\mathrm{R}=4-\mathrm{OCH}_{3}-\mathrm{C}_{6} \mathrm{H}_{4}$

10g, $\mathrm{R}=-\mathrm{CH}_{2} \mathrm{C}_{6} \mathrm{H}_{4}$

10h, $\mathrm{R}=\mathrm{CH}_{3}$

$10 i, R=$<smiles>[R]CC(C)=CC1=CC=CC(=CC)C=C1</smiles>

(iii)<smiles>[R]N([O-])S(=O)(=O)c1ccccc1N</smiles>

9b, $\mathrm{R}=4-\mathrm{Cl}-\mathrm{C}_{6} \mathrm{H}_{4}$ (v)<smiles>CCOC(=O)N(c1ccc(Cl)cc1)S(=O)(=O)c1ccccc1N</smiles>

$9 b$ (iv)<smiles>CCOC(=O)N(c1ccc(Cl)cc1)S(=O)(=O)c1ccccc1N=Pc1ccccc1</smiles>

12b

Scheme 2: Preparation of 3-ethoxy-1,2,4-benzothiadiazine 1,1-dioxide. Reagent and conditions: (i) $\left(\mathrm{COCl}_{2}, \mathrm{DMF} \mathrm{CH}_{2} \mathrm{Cl}_{2}, \mathrm{reflux}, 3 \mathrm{~h}\right.$; (ii) $\mathrm{RNH}$, $\mathrm{NaOAc}, \mathrm{MeOH}+$ water, $60^{\circ} \mathrm{C}$; (iii) $\mathrm{ClCO}_{2} \mathrm{C}_{2} \mathrm{H}_{5}$, acetone, $\mathrm{Et}_{3} \mathrm{~N}$, rt, $5 \mathrm{~h}$; (iv) $\mathrm{PPh}_{3}$, THF, reflux, $10 \mathrm{~h}$; (v) $\mathrm{PPh}_{3}, \mathrm{DCB}, 135^{\circ} \mathrm{C}, 8 \mathrm{~h}$.

Table 1: Summary of the intramolecular aza-Wittig reactions. ${ }^{a}$<smiles>CCOC(=O)N(c1ccc(Cl)cc1)S(=O)(=O)c1ccccc1N</smiles>

$9 \mathbf{b}$<smiles>COC(=O)N(c1ccc(Cl)cc1)c1ccccc1S(=O)(=O)N(C)c1ccccc1</smiles>

$12 b$<smiles>CCOC1=Nc2ccccc2S(=O)(=O)N1c1ccc(Cl)cc1</smiles>

$13 b$

\begin{tabular}{lllll}
\hline Entry & Solvent & Temp $\left({ }^{\circ} \mathrm{C}\right)$ & Time $(\mathrm{h})$ & Yield $(\%)^{\mathrm{b}}$ \\
\hline $1^{\mathrm{C}}$ & $\mathrm{THF}$ & reflux & 6 & 0 \\
$2^{\mathrm{C}}$ & toluene & $120^{\circ} \mathrm{C}$ & 8 & 0 \\
$3^{\mathrm{C}}$ & $\mathrm{CH}_{2} \mathrm{Cl} 2$ & reflux & 8 & 0 \\
$4^{\mathrm{C}}$ & $\mathrm{CH}_{3} \mathrm{CN}$ & reflux & 6 & 0 \\
5 & $\mathrm{DCB}$ & $135^{\circ} \mathrm{C}$ & 8 & 54 \\
\hline
\end{tabular}

${ }^{\mathrm{a}} \mathrm{All}$ the reactions were carried out with 1 equiv $9 \mathrm{~b}$ and 1.5 equiv $\mathrm{PPh}_{3}$; ${ }^{\mathrm{b}}$ isolated yields of $13 \mathbf{b}$; ${ }^{\mathrm{c}}$ only $\mathbf{1 2 b}$ was separated. 
Table 2: Summary of the intramolecular aza-Wittig reactions in a one-pot fashion. ${ }^{a}$<smiles>N#Cc1ccccc1S(=O)(=O)Nc1ccc(Cl)cc1</smiles>

$10 \mathrm{~b}$<smiles>CCOC1=Nc2ccccc2S(=O)(=O)N1c1ccc(Cl)cc1</smiles>

$13 b$<smiles>CCOC(N)c1ccc(Cl)cc1</smiles>

$14 b$

\begin{tabular}{|c|c|c|c|c|c|}
\hline Entry & Solvent & Base & Temp $\left({ }^{\circ} \mathrm{C}\right)$ & Time (h) & Yield $(\%)^{b}$ \\
\hline 1 & DCB & $\mathrm{Et}_{3} \mathrm{~N}$ & $135^{\circ} \mathrm{C}$ & 6 & 78 \\
\hline 2 & $\mathrm{DCB}$ & $\mathrm{K}_{2} \mathrm{CO}_{3}$ & $135^{\circ} \mathrm{C}$ & 8 & $<30$ \\
\hline 3 & DCB & $\mathrm{Cs}_{2} \mathrm{CO}_{3}$ & $135^{\circ} \mathrm{C}$ & 8 & 46 \\
\hline 4 & $\mathrm{DCB}$ & DIPEA & $135^{\circ} \mathrm{C}$ & 6 & 69 \\
\hline 5 & xylene & $\mathrm{Et}_{3} \mathrm{~N}$ & $135^{\circ} \mathrm{C}$ & 6 & 94 \\
\hline 6 & xylene & DIPEA & $135^{\circ} \mathrm{C}$ & 6 & 92 \\
\hline $7^{c}$ & xylene & $\mathrm{Et}_{3} \mathrm{~N}$ & $150^{\circ} \mathrm{C}$ & 6 & 5 \\
\hline 8 & xylene & - & $135^{\circ} \mathrm{C}$ & 10 & 0 \\
\hline
\end{tabular}

${ }^{a}$ All the reactions were carried out with 1 equiv $10 b, 1.5$ equiv $\mathrm{ClCO}_{2} \mathrm{Et}, 2$ equiv base, and 1.5 equiv $\mathrm{PPh}_{3}$; ${ }^{\mathrm{b}}$ isolated yields of $13 \mathbf{b}$; ${ }^{\mathrm{c} a}$ smaller amount of $13 \mathrm{~b}$ was isolated than the major product $14 \mathrm{~b}$.

yield of the product (Table 2, entry 6). The decomposition of the iminophosphorane intermediate into the corresponding amine derivative 14b was found to occur at higher temperature $\left(150{ }^{\circ} \mathrm{C}\right.$ ) producing a low yield of the cyclized product (Table 2 , entry 7). The reaction did not occur at all in the absence of a base (Table 2, entry 8). The observations are summarized in Table 2.
It is notable that xylene appears to be a suitable solvent for this reaction. We then carried out the reactions with a variety of substrates 10a-i under the optimized conditions (ethyl carbonochloridate, $\mathrm{PPh}_{3}, \mathrm{Et}_{3} \mathrm{~N}$, xylene at $135^{\circ} \mathrm{C}$ ) in order to generalize the method, and the results are summarized in Table 3. The reactions of all the substrates having electron-deficient R-substituents at the 2-position proceeded smoothly,

Table 3: Generalization of intramolecular aza-Wittig reaction. ${ }^{a}$<smiles>[R]NS(=O)(=O)c1ccccc1N</smiles>

10<smiles>[R]N1C(OCC)=Nc2ccccc2S1(=O)=O</smiles>

13

\begin{tabular}{|c|c|c|c|c|}
\hline Entry & o-azidosulfonamide & Time (h) & Product & Yield $(\%)^{b}$ \\
\hline 1 & $10 \mathbf{a}, \mathrm{R}=\mathrm{C}_{6} \mathrm{H}_{5}$ & 8 & 13a, $\mathrm{R}=\mathrm{C}_{6} \mathrm{H}_{5}$ & 90 \\
\hline 2 & $10 b, \mathrm{R}=4-\mathrm{Cl}-\mathrm{C}_{6} \mathrm{H}_{4}$ & 6 & $13 b, \mathrm{R}=4-\mathrm{Cl}-\mathrm{C}_{6} \mathrm{H}_{4}$ & 94 \\
\hline 3 & $10 c, R=4-\mathrm{COCH}_{3}-\mathrm{C}_{6} \mathrm{H}_{4}$ & 6 & $13 \mathrm{c}, \mathrm{R}=4-\mathrm{COCH}_{3}-\mathrm{C}_{6} \mathrm{H}_{4}$ & 92 \\
\hline 4 & $10 \mathrm{~d}, \mathrm{R}=4-\mathrm{CO}_{2} \mathrm{CH}_{3}-\mathrm{C}_{6} \mathrm{H}_{4}$ & 6 & 13d, $\mathrm{R}=4-\mathrm{CO}_{2} \mathrm{CH}_{3}-\mathrm{C}_{6} \mathrm{H}_{4}$ & 95 \\
\hline 5 & $10 e, \mathrm{R}=4-\mathrm{CH}_{3}-\mathrm{C}_{6} \mathrm{H}_{4}$ & 7 & $13 e, \mathrm{R}=4-\mathrm{CH}_{3}-\mathrm{C}_{6} \mathrm{H}_{4}$ & 80 \\
\hline 6 & 10f, $\mathrm{R}=4-\mathrm{OCH}_{3}-\mathrm{C}_{6} \mathrm{H}_{4}$ & 7 & 13f, $\mathrm{R}=4-\mathrm{OCH}_{3}-\mathrm{C}_{6} \mathrm{H}_{4}$ & 83 \\
\hline 7 & $10 \mathrm{~g}, \mathrm{R}=-\mathrm{CH}_{2} \mathrm{C}_{6} \mathrm{H}_{5}$ & 7 & $13 g, R=-\mathrm{CH}_{2} \mathrm{C}_{6} \mathrm{H}_{5}$ & 87 \\
\hline 8 & $10 h, \mathrm{R}=\mathrm{CH}_{3}$ & 7 & $13 h, \mathrm{R}=\mathrm{CH}_{3}$ & 79 \\
\hline 9 & $10 i, R=$ & 6 & $13 i, R=$ & 89 \\
\hline
\end{tabular}

aReaction conditions: Compound $10(1 \mathrm{mmol}), \mathrm{ClCO}_{2} \mathrm{C}_{2} \mathrm{H}_{5}(1.5 \mathrm{mmol}), \mathrm{Et}_{3} \mathrm{~N}(2 \mathrm{mmol})$ and $\mathrm{PPh}_{3}(1.5 \mathrm{mmol})$ were heated at $135{ }^{\circ} \mathrm{C}$ in xylene; bisolated yields of compound 13. 


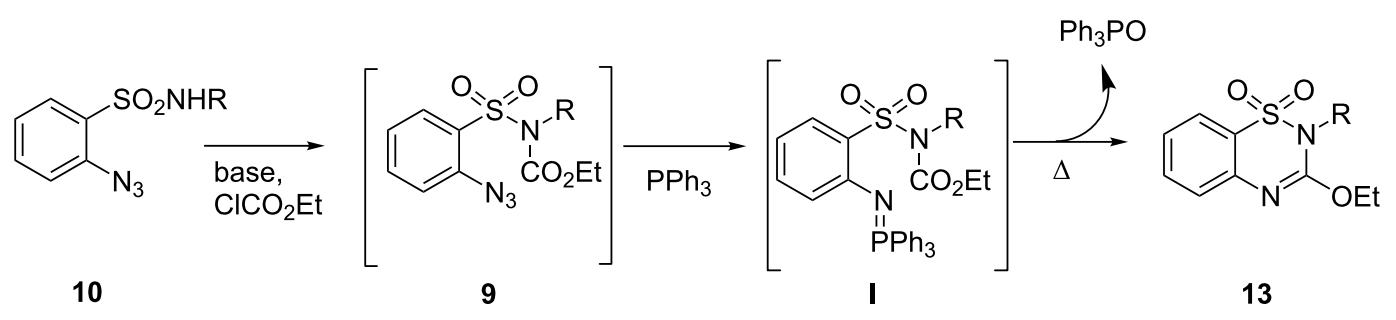

Scheme 3: Rationalization of the formation of compound 13

providing excellent yields, whereas the substrates having electron-donating R-substituents gave lower yields.

The proposed mechanism for the formation of the products $\mathbf{1 3}$ may involve amidation of $\mathrm{SO}_{2} \mathrm{NH}_{2}$ by the reaction of nucleophilic sulfonamide $\mathbf{1 0}$ with ethyl carbonochloridate in the presence of $\mathrm{Et}_{3} \mathrm{~N}$ to form the intermediates 9, which may then undergo intramolecular aza-Wittig reaction via the formation of iminophosphorane intermediate $\mathbf{I}$. We isolated iminophosphorane intermediate $\mathbf{1 2 b}$ from the reaction with $\mathbf{1 0 b}$ at room temperature. In the presence of heat the iminophosphorane intermediate I leads to the formation of the product 3-ethoxy1,2,4-benzothiadiazine 1,1-dioxide 13 (Scheme 3). However, in all other cases we did not carry out the reactions at room temperature for isolation of the intermediates.

We have also demonstrated the conversion of the products $\mathbf{1 3}$ to the 2 -substituted benzothiadiazine-3-one 1,1-dioxide $\mathbf{1 5}$ by hydrolysing 13 with ethanolic $\mathrm{HCl}$. The benzothiadiazine-3-one 1,1-dioxide derivatives $\mathbf{1 5 c}, \mathbf{e}, \mathbf{h}$ were obtained in excellent yields from the compounds $\mathbf{1 3 c}, \mathbf{e}, \mathbf{h}$ (Scheme 4). These 2-substituted benzothiadiazine-3-one 1,1-dioxides may further be alkylated at the 4-position with suitable halides to yield the RSV inhibitors 5 and 6 by using the reported [13] procedure.

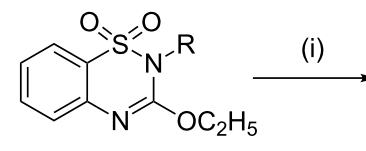

$$
\begin{aligned}
& \overbrace{}^{O} \\
& \text { 13h, } \mathrm{R}=\mathrm{CH}_{3} \quad 15 \mathrm{~h}, \mathrm{R}=\mathrm{CH}_{3} \text {, Yield: } 97 \%
\end{aligned}
$$

Scheme 4: Preparation of benzothiadiazine-3-one 1,1-dioxide derivatives by acid-catalyzed hydrolysis; reagents and conditions: $50 \mathrm{mg}$ of compound $13,1 \mathrm{~mL} \mathrm{HCl}, 4 \mathrm{~mL}$ ethanol, $80^{\circ} \mathrm{C}, 4 \mathrm{~h}$.

Previously, Jung and Khazi [52] reported the synthesis of the benzothiadiazine 1,1-dioxide moiety from the reaction of $o$-aminobenzenesulfonamide with the costlier triphosgene, whereas in our case the synthesis of benzothiadiazine 1,1- dioxide derivatives was achieved from $o$-azidobenzenesulfonamides and required cheaper ethyl carbonochloridate as the reagent.

\section{Conclusion}

In conclusion, we have developed a simple and efficient method for the synthesis of 3-ethoxybenzothiadiazine 1,1-dioxide and benzothiadiazine-3-one 1,1-dioxide derivatives starting from an easy precursor, by the application of an intramolecular azaWittig reaction. The reaction procedure is very simple and gives good to excellent yields of the products. This benzothiadiazine3-one 1,1-dioxide can further be alkylated at the 4-position, following a literature procedure, to give the bioactive RSV inhibitors.

\section{Supporting Information}

\section{Supporting Information File 1}

Experimental part.

[http://www.beilstein-journals.org/bjoc/content/ supplementary/1860-5397-9-54-S1.pdf]

\section{Acknowledgements}

We thank CSIR (New Delhi) and DST (New Delhi) for financial assistance. S.G. is grateful to CSIR (New Delhi) for his research fellowships, and K.C.M. is thankful to UGC (New Delhi) for a UGC Emeritus fellowship. We also thank DST (New Delhi) for providing the Bruker NMR (400 MHz), the Perkin-Elmer CHN Analyser, and the FTIR and UV-vis spectrometers.

\section{References}

1. Majumdar, K. C.; Mondal, S. Chem. Rev. 2011, 111, 7749-7773. doi:10.1021/cr1003776

2. Bernotas, R. C.; Dooley, R. J. Tetrahedron 2010, 66, 2273-2276. doi:10.1016/j.tet.2010.01.092

3. Zhou, A.; Rayabarapu, D.; Hanson, P. R. Org. Lett. 2009, 11, 531-534. doi:10.1021/ol802467f

4. Jiménez-Hopkins, M.; Hanson, P. R. Org. Lett. 2008, 10, 2223-2226. doi:10.1021/ol800649n 
5. Supuran, C. T.; Casini, A.; Scozzafava, A. Med. Res. Rev. 2003, 23, 535-558. doi:10.1002/med.10047

6. Hanessian, S.; Sailes, H.; Therrien, E. Tetrahedron 2003, 59 , 7047-7056. doi:10.1016/S0040-4020(03)00919-0

7. Dauban, P.; Dodd, R. H. Tetrahedron Lett. 2001, 42, 1037-1040. doi:10.1016/S0040-4039(00)02214-0

8. Drews, J. Science 2000, 287, 1960-1964 doi:10.1126/science.287.5460.1960

9. Wales, J. K.; Krees, S. V.; Grant, A. M.; Vikroa, J. K.; Wolff, F. W. J. Pharmacol. Exp. Ther. 1968, 164, 421-432.

10. Scozzofava, A.; Owa, T.; Mastrolorenzo, A.; Supuran, C. T. Curr. Med. Chem. 2003, 10, 925-953. doi:10.2174/0929867033457647

11. Casini, A.; Scozzafava, A.; Mastrolorenco, A.; Supuran, C. T. Curr. Cancer Drug Targets 2002, 2, 55-75. doi:10.2174/1568009023334060

12. Scozzafava, A.; Casini, A.; Supuran, C. T. Curr. Med. Chem. 2002, 9, 1167-1185. doi:10.2174/0929867023370077

13. Arranz, E. M.; Díaz, J. A.; Ingate, S. T.; Witvrouw, M.; Pannecouque, C.; Balzarini, J.; De Clercq, E.; Vega, S. Bioorg. Med. Chem. 1999, 7, 2811-2822. doi:10.1016/S0968-0896(99)00221-7

14. Wang, Y.; Busch-Petersen, J.; Wang, F.; Ma, L.; Fu, W.; Kerns, J. K.; Jin, J.; Palovich, M. R.; Shen, J.-K.; Burman, M.; Foley, J. J.; Schmidt, D. B.; Hunsberger, G. E.; Sarau, H. M.; Widdowson, K. L. Bioorg. Med. Chem. Lett. 2007, 17, 3864-3867. doi:10.1016/j.bmcl.2007.05.011

15. Hayao, S.; Stryker, W.; Phillips, B.; Fujimori, H.; Vidrio, H. J. Med. Chem. 1968, 11, 1246-1248. doi:10.1021/jm00312a601

16. Khelili, S.; Kihal, N.; Yekhlef, M.; de Tullio, P.; Lebrun, P.; Pirotte, B. Eur. J. Med. Chem. 2012, 54, 873-878. doi:10.1016/j.ejmech.2012.05.011

17. de Tullio, P.; Servais, A.-C.; Fillet, M.; Gillotin, F.; Somers, F.; Chiap, P.; Lebrun, P.; Pirotte, B. J. Med. Chem. 2011, 54, 8353-8361. doi:10.1021/jm200786z

18. Francotte, P.; Goffin, E.; Fraikin, P.; Lestage, P.; van Heugen, J.-C.; Gillotin, F.; Danober, L.; Thomas, J.-Y.; Chiap, P.; Caignard, D.-H.; Pirotte, B.; de Tullio, P. J. Med. Chem. 2010, 53, 1700-1711. doi:10.1021/jm901495t

19. Pirotte, B.; de Tullio, P.; Nguyen, Q.-A.; Somers, F.; Fraikin, P.; Florence, X.; Wahl, P.; Hansen, J. B.; Lebrun, P. J. Med. Chem. 2010, 53, 147-154. doi:10.1021/jm9010093

20. Francotte, P.; de Tullio, P.; Goffin, E.; Dintilhac, G.; Graindorge, E.; Fraikin, P.; Lestage, P.; Danober, L.; Thomas, J.-Y.; Caignard, D.-H.; Pirotte, B. J. Med. Chem. 2007, 50, 3153-3157. doi:10.1021/jm070120i

21. Combrink, K. D.; Gulgeze, H. B.; Thuring, J. W.; Yu, K.-L.; Civiello, R. L.; Zhang, Y.; Pearce, B. C.; Yin, Z.; Langley, D. R.; Kadow, K. F.; Cianci, C. W.; Li, Z.; Clarke, J.; Genovesi, E. V.; Medina, I.; Lamb, L.; Yang, Z.; Zadjura, L.; Krystal, M.; Meanwell, N. A. Bioorg. Med. Chem. Lett. 2007, 17, 4784-4790. doi:10.1016/j.bmcl.2007.06.065

22. Boverie, S.; Antoine, M.-H.; Somers, F.; Becker, B.; Sebille, S.; Ouedraogo, R.; Counerotte, S.; Pirotte, B.; Lebrun, P.; de Tullio, P. J. Med. Chem. 2005, 48, 3492-3503. doi:10.1021/jm0311339

23. Girard, Y.; Atkinson, J. G.; Rokach, J. J. Chem. Soc., Perkin Trans. 1 1979, 1043-1047. doi:10.1039/P19790001043

24. Chern, J.-W.; Ho, C.-P.; Wu, Y.-H.; Rong, J.-G.; Liu, K.-C.; Cheng, M.-C.; Wang, Y. J. Heterocycl. Chem. 1990, 27, 1909-1915. doi:10.1002/jhet.5570270712

25. Cherepakha, A.; Kovtunenko, V. O.; Tolmachev, A.; Lukin, O. Tetrahedron 2011, 67, 6233-6239. doi:10.1016/j.tet.2011.06.063
26. Hirota, S.; Sakai, T.; Kitamura, N.; Kubokawa, K.; Kutsumura, N.; Otani, T.; Saito, T. Tetrahedron 2010, 66, 653-662. doi:10.1016/j.tet.2009.11.064

27. Yang, D.; Liu, H.; Yang, H.; Fu, H.; Hu, L.; Jiang, Y.; Zhao, Y. Adv. Synth. Catal. 2009, 351, 1999-2004. doi:10.1002/adsc.200900101

28. Rolfe, A.; Hanson, P. R. Tetrahedron Lett. 2009, 50, 6935-6937. doi:10.1016/j.tetlet.2009.09.090

29. Hirota, S.; Kato, R.; Suzuki, M.; Soneta, Y.; Otani, T.; Saito, T. Eur. J. Org. Chem. 2008, 2075-2083. doi:10.1002/ejoc.200701131

30. Blackburn, C.; Achab, A.; Elder, A.; Ghosh, S.; Guo, J.; Harriman, G.; Jones, M. J. Org. Chem. 2005, 70, 10206-10209. doi:10.1021/jo051843h

31. Su, W.; Cai, H.; Yang, B. J. Chem. Res. 2004, 87-88. doi:10.3184/030823404323000936

32. Makino, S.; Nakanishi, E.; Tsuji, T. J. Comb. Chem. 2003, 5, 73-78. doi:10.1021/cc020056k

33. Xie, H.; Yuan, D.; Ding, M.-W. J. Org. Chem. 2012, 77, 2954-2958. doi:10.1021/jo202588j

34. Zhong, Y.; Wang, L.; Ding, M.-W. Tetrahedron 2011, 67, 3714-3723. doi:10.1016/j.tet.2011.03.056

35. Palacios, F.; Alonso, C.; Aparicio, D.; Rubiales, G.; de los Santos, J. M. Tetrahedron 2007, 63, 523-575. doi:10.1016/j.tet.2006.09.048

36. Cossío, F. P.; Alonso, C.; Lecea, B.; Ayerbe, M.; Rubiales, G.; Palacios, F. J. Org. Chem. 2006, 71, 2839-2847. doi:10.1021/jo0525884

37. Palacios, F.; Aparicio, D.; Rubiales, G.; Alonso, C.; de los Santos, J. M. Curr. Org. Chem. 2006, 10, 2371-2392. doi:10.2174/138527206778992716

38. Eguchi, S. Top. Heterocycl. Chem. 2006, 6, 113-156. doi:10.1007/7081_022

39. Cassidy, M. P.; Özdemir, A. D.; Padwa, A. Org. Lett. 2005, 7, 1339-1342. doi:10.1021/ol0501323

40. Snider, B. B.; Zhon, J. J. Org. Chem. 2005, 70, 1087-1088. doi:10.1021/jo048131w

41. Gil, C.; Bräse, S. Chem.-Eur. J. 2005, 11, 2680-2688. doi:10.1002/chem.200401112

42. Alajarín, M.; Sánchez-Andrada, P.; Vidal, A.; Tovar, F. J. Org. Chem. 2005, 70, 1340-1349. doi:10.1021/jo0482716

43. Fresneda, P. M.; Molina, P. Synlett 2004, 1-17. doi:10.1055/s-2003-43338

44. Majumdar, K. C.; Ray, K.; Ganai, S. Synlett 2010, 2122-2124. doi:10.1055/s-0030-1258519

45. Majumdar, K. C.; Ganai, S.; Sinha, B. Tetrahedron 2012, 68 , 7806-7811. doi:10.1016/j.tet.2012.07.040

46. Majumdar, K. C.; Ganai, S.; Nandi, R. K.; Ray, K. Tetrahedron Lett. 2012, 53, 1553-1557. doi:10.1016/j.tetlet.2012.01.015

47. Majumdar, K. C.; Ganai, S.; Nandi, R. K. New J. Chem. 2011, 35, 1355-1359. doi:10.1039/c1nj20121b

48. Majumdar, K. C.; Ganai, S.; Chattopadhyay, B.; Ray, K. Synlett 2011, 2369-2373. doi:10.1055/s-0030-1260312

49. Majumdar, K. C.; Ganai, S. Synlett 2011, 1881-1887. doi:10.1055/s-0030-1260975

50. Majumdar, K. C.; Ray, K.; Ganai, S.; Ghosh, T. Synthesis 2010, 858-862. doi:10.1055/s-0029-1218610

51. Majumdar, K. C.; Ray, K.; Ganai, S. Synthesis 2010, 2101-2105. doi:10.1055/s-0029-1218763

52. Khazi, I. A.; Jung, Y.-S. Lett. Org. Chem. 2007, 4, 423-428. doi:10.2174/157017807781467641 


\section{License and Terms}

This is an Open Access article under the terms of the Creative Commons Attribution License

(http://creativecommons.org/licenses/by/2.0), which permits unrestricted use, distribution, and reproduction in any medium, provided the original work is properly cited.

The license is subject to the Beilstein Journal of Organic Chemistry terms and conditions:

(http://www.beilstein-journals.org/bjoc)

The definitive version of this article is the electronic one which can be found at:

doi:10.3762/bjoc.9.54 